BNL 956 (T-397) 38

5

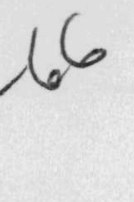

BROOKHAVEN LECTURE SERIES

\title{
Symmetry Principles in Physics
}

C.N. YANG

Institute for Advanced Study, Princeton

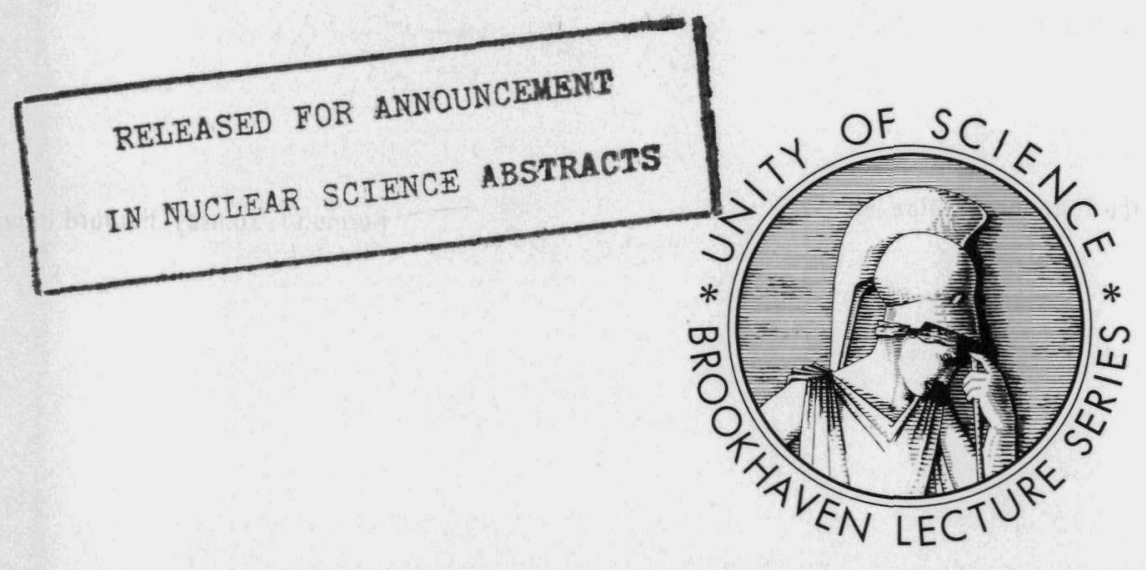

Number 50

October 13, 1965

\section{BROOKHAVEN NATIONAL LABORATORY}

Associated Universities, Inc.

under contract with the

United States Atomic Energy Commission 


\section{DISCLAIMER}

Portions of this document may be illegible in electronic image products. Images are produced from the best available original document. 


\section{FOREWORD}

The Brookhaven Lectures, held by and for the Brookhaven staff, are meant to provide an intellectual meeting ground for all scientists of the Laboratory. In this role they serve a double purpose: they are to acquaint the listeners with new developments and ideas not only in their own field, but also in other important fields of science, and to give them a heightened awareness of the aims and potentialities of Brookhaven National Laboratory.

Before describing some recent research or the novel design and possible uses of a machine or apparatus, the lecturers attempt to familiarize the audience with the background of the topic to be treated and to define unfamiliar terms as far as possible.

Of course we are fully conscious of the numerous hurdles and pitfalls which necessarily beset such a venture. In particular, the difference in outlook and method between physical and biological sciences presents formidable difficulties. However, if we wish to be aware of progress in other fields of science, we have to consider each obstacle as a challenge which can be met.

The lectures are found to yield some incidental rewards which heighten their spell: In order to organize his talk the lecturer has to look at his work with a new, wider perspective, which provides a satisfying contrast to the often very specialized point of view from which he usually approaches his theoretical or experimental research. Conversely, during the discussion period after his talk, he may derive valuable stimulation from searching questions or technical advice received from listeners with different scientific backgrounds. The audience, on the other hand, has an opportunity to see a colleague who may have long been a friend or acquaintance in a new and interesting light.

The lectures are being organized by a committee which consists of representatives of all departments of the Laboratory. A list of the lectures that have been given and of those which are now scheduled appears on the back of this report.

Gertrude Scharff-Goldhaber

The drawing on the cover is taken from a 5th Century B.C. relief on the Acropolis in Athens, the "Dreaming Athena," by an unknown sculptor. 


\title{
Symmetry Principles in Physics
}

\author{
C.N. YANG
}

7i.C. $\$ 1.00 ; \mathrm{MN}, 50$

Institute for Advanced Study, Princeton

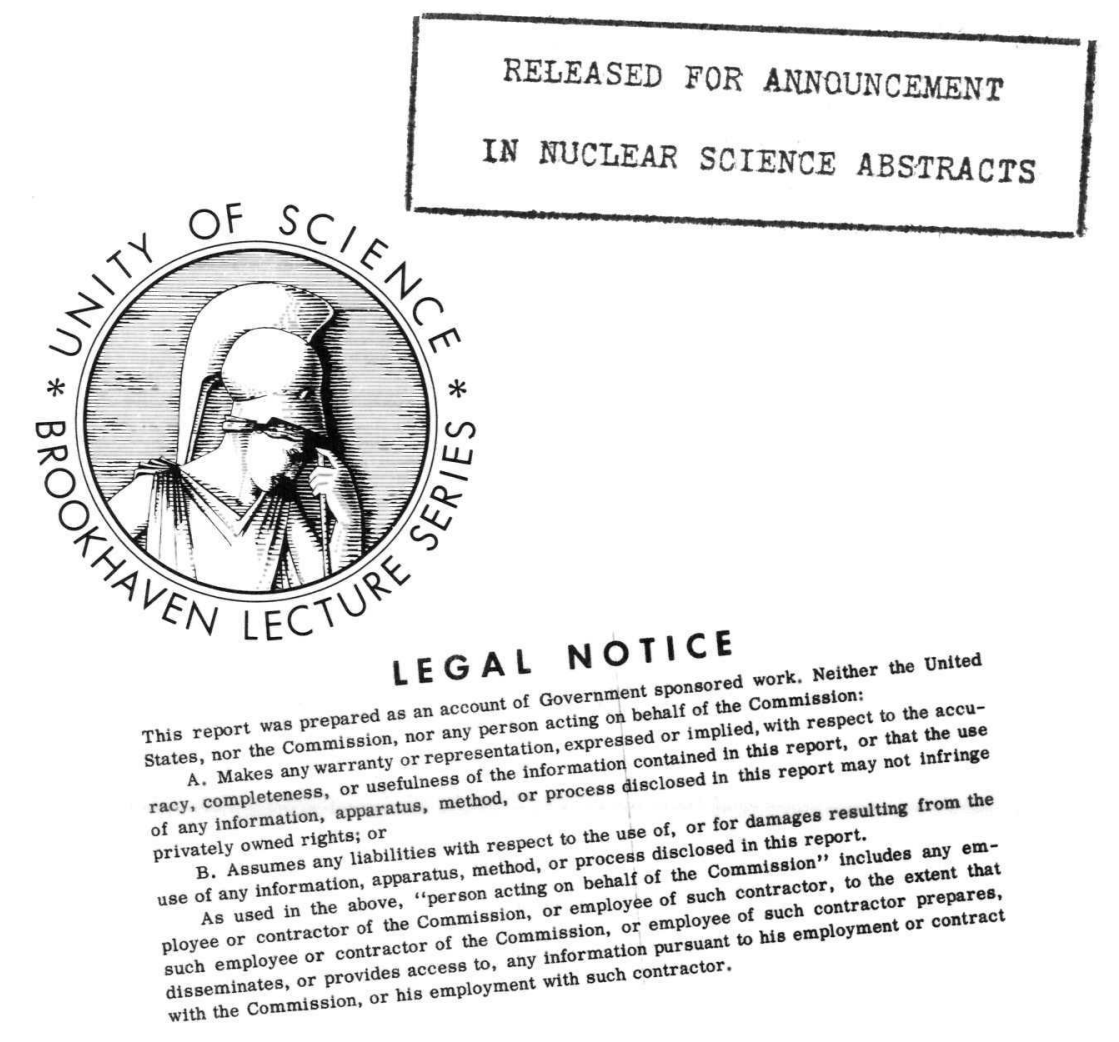

\section{BROOKHAVEN LECTURE SERIES}

Number 50, October 13, 1965 


\section{E G A L NOT I C E}

This report was prepared as an account of Government sponsored work. Neither the United States, nor the Commission, nor any person acting on behalf of the Commission:

A. Makes any warranty or representation, expressed or implied, with respect to the accuracy, completeness, or usefulness of the information contained in this report, or that the use of any information, apparatus, method, or process disclosed in this report may not infringe privately owned rights; or

B. Assumes any liabilities with respect to the use of, or for damages resulting from the use of any information, apparatus, method, or process disclosed in this report.

As used in the above, "person acting on behalf of the Commission" includes any employee or contractor of the Commission, or employee of such contractor, to the extent that such employee or contractor of the Commission, or employee of such contractor prepares, disseminates, or provides access to, any information pursuant to his employment or contract with the Commission, or his employment with such contractor.

\section{PRINTED IN USA}

\section{PRICE $\$ 1.00$}

Available from the

Clearinghouse for Federal Scientific and Technical Information

National Bureau of Standards

U.S. Department of Commerce

Springfield, Virginia 


\section{INTRODUCTION}

We did not have to stretch the definition of the Brookhaven Lectures very much to be able to invite tonight's speaker. These lectures are supposed to be held by and for the Brookhaven staff. We cannot help thinking of tonight's speaker as one of our own.

Chen Ning Yang was born in China, the son of a professor of mathematics. He received his college education at the National Southwest Associated University in Kunming, China, and completed his B.Sc. degree there in 1942. His M.Sc. degree was received in 1944 from Tsinghua University, which had moved to Kunming during the Sino-Japanese War (1937-1945). He went to the U.S.A. at the end of the war on a Tsinghua University Fellowship, and entered the University of Chicago in January 1946. There he assumed the name Frank or Franklin out of admiration for President Roosevelt, I assume. At Chicago he studied with Fermi and Teller. After receiving his Ph.D. degree in 1948, Yang served for a year at the University of Chicago as an instructor. With Fermi he introduced the idea that the $\pi$ meson may be composed of a nucleon and an antinucleon, an idea which has led to many further fruitful generalizations of which we shall hear later. He has been associated with the Institute for Advanced Study at Princeton since 1949, where he became a professor in 1955. Frank Yang has worked on various subjects in physics, but his chief interest is in two fields: statistical mechanics and symmetry principles.

Frank Yang has received many honors, including the Nobel Prize which he shared with T.D. Lee in 1957 for asking the famous question, "Is parity conserved in weak interactions?" This important work was written at Brookhaven in the summer of 1956. When the experiment answered with "No," it was not too hard to explain to the general public that left is different from right. They always knew that. Recently, an experiment done at the AGS by Christenson, Cronin, Fitch, and Turlay of Princeton has thrown some doubt on another symmetry principle, namely, time reversal. Again it will not be too difficult to explain to the man in the street that the future is not like the past. He has always known that.

Frank Yang and his family live in Princeton but we expect that by next year he will become a close neighbor when he joins the State University of New York at Stony Brook. I am very happy to introduce Frank Yang.

M. Goldhaber 


\section{Symmetry Principles in Physics}

About 120 years ago, at the very beginning of his scientific career, Pasteur made a great discovery that enormously excited him and the chemists of his time. The discovery was to lead to the concept of the existence of a new kind of isomerism in chemistry. It was to open the way to a new branch of science called stereochemistry. What Pasteur found was that a certain racemic salt, which was known to be optically inactive, when crystallized from a water solution at low temperatures, possessed two kinds of crystals which were nonsuperposable mirror images of each other. (See Figure 1.) Pasteur separated with infinite pains under the microscope the two different crystal forms and thereupon came to the discovery that one of them was precisely a known organic compound - of tartaric acid - which was optically active, and the other was a hitherto unknown compound with the opposite optical activity. Some 70 years later, Jaegar, Professor of Chemistry at the University of Groningen in the Netherlands, in his lecture on "The Principle of Symmetry" remarked, "seldom has a scientific discovery had such far-reaching consequences as this one had." In particular, the discovery made such a profound impression on Pasteur himself that he came back repeatedly to the problem of the separation of optically inactive substances into optically active components in later years. As a matter of fact, about 10 years after his first discovery he found that a certain type of plant mold could also effect the separation of the racemic acid. He then reflected on the fact that no ordinary chemical agents or heat could cause the separation and that the two separation methods known to him were both via living agents: in the one case via the mold, in the other case via Pasteur himself. He became convinced that the ability to effect such a separation was "the prerogative of life." This view led to heated discussions, especially after a famous lecture by Japp in 1898 entitled "Stereochemistry and Vitalism" which was published in Nature $^{1}$ and was the presidential address in the chemistry section of the British Association. He argued for a new version of vitalism in the origin of life. He said, "We thus arrive at the conclusion that the production of single asymmetrical compounds, or their isolation ... ., is, as Pasteur firmly held, the prerogative of life. Only the living organism with its asym- metrical tissues,or the asymmetrical products of the living organism, or the living intelligence with its conception of asymmetry, can produce this result. Only asymmetry can beget asymmetry."

Leaving aside the question of vitalism, we know today, of course, that the two crystalline forms of tartaric acid are manifestations of their different atomic arrangements. The two elementary molecular forms are mirror images of each other. (See Figure 2.) We also know that most complicated organic molecules are not equivalent to their mirror images. Prevalence of one mirror image form over the other, as, e.g., exhibited in the structure of the DNA molecule (see Figure 3), is at the root of the ability for organic material to create optical activity.

The fascination that Pasteur and his contemporaries showed in the question of the symmetry of molecules is hardly surprising. For in natural phenomena, and in various fields of human activity, symmetry has always played an important role. We have beautiful illustrations in Figure 4 of marine creatures exhibiting almost perfect octahedral, icosahedral, and dodecahedral symmetries. In human activities - in music, in literature, in mathematics, and in art - symmetry oftentimes forms the basic element of esthetic appeal. Figure 5 shows a view of a huge Chinese bronzeware dating back to some 3300 years ago. What motivated the artist to such weird, ferocious, and symmetrical forms the art historians have not yet learned.

In physics the concept of symmetry has been naturally incorporated since the beginning of the science. With the discussion of crystalline structure, symmetry considerations gained new prominence. However, it was only with the introduction of quantum mechanics that symmetry principles became a part of the language of physics. The use of quantum numbers and selection and intensity rules (Figure 6) made symmetry considerations the starting point of much of atomic, molecular, nuclear, and elementary particle physics. One could ask for the reason behind the vastly different roles played by symmetry in classical and in quantum physics. The reason is not hard to find. The superposition principle in quantum mechanics allows symmetry considerations to be applied to all forms 

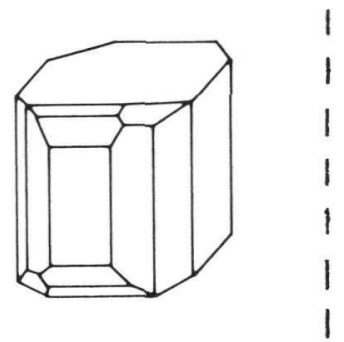

Figure 1. Two crystal forms of sodium ammonium tartrate. The two forms are mirror images of each other, but are not superimposable. [From Kurt Mislow, Introduction to Stereochemistry, p. 53, W.A. Benjamin, Inc., 1965.]<smiles>O=C(O)C(O)C(=O)O</smiles>

$A$<smiles>O=C(O)C1CC2CC(C(=O)O)C(C2O)C1O</smiles>

B

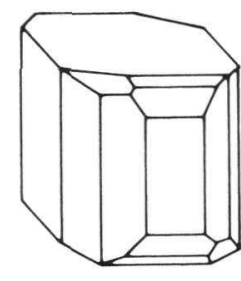

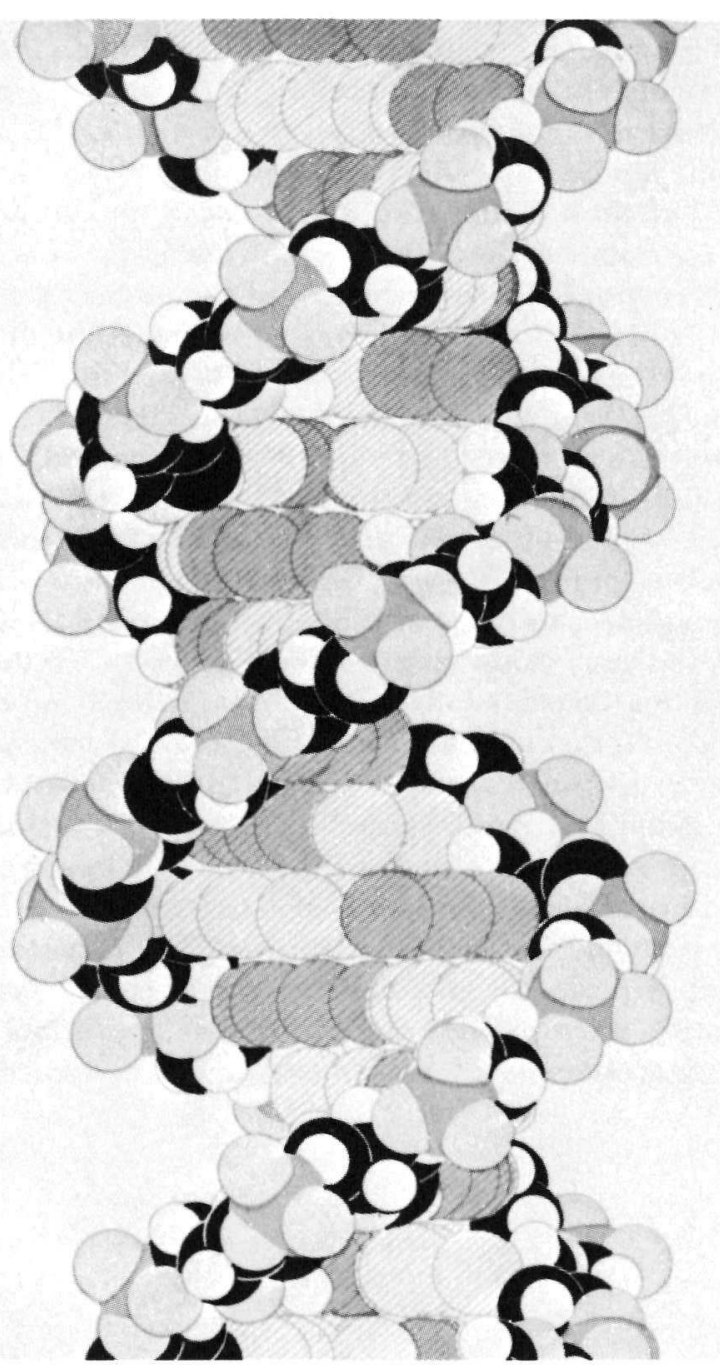

Figure 2. Molecular structure of two kinds of tartaric acid. The C-C bonds in $A$ and $\bar{A}$ are in the plane of the page. Wedge-shaped bonds stick upwards from the page; dashed bonds stick downwards into the page. $B$ is a view of $A$ along the C-C bond. $\bar{B}$ is a view of $B$ along the C-C bond. $A$ and $\bar{A}$ are nonsuperposable mirror images of each other. [From Kurt Mislow, Introduction to Stereochemistry, p. 28, W.A. Benjamin, Inc., 1965.]

of motion (e.g., elliptical as well as circular orbits), and it allows symmetry considerations to be formulated in terms of the theory of group representations, a powerful mathematical concept of great beauty and depth.

The enormous effectiveness of symmetry considerations in quantum mechanics naturally led to a proliferation of papers concerning the subject, especially in recent years. Table 1 illustrates six different types of symmetry considerations that are currently being written about in elementary particle physics. We shall discuss the first two types in some detail. For the other types, let me only mention that one of the most useful symmetry concepts in elementary particle physics is isotopic spin invariance, designated $\mathbf{I}$ in the table. While the existence of such a symmetry is dramatically demonstrated simply by a glance at the masses of the elementary particles (Figure 7), there is today only a descriptive understanding of the origin of this symmetry. The point I want to make here can be illustrated by the following fictitious example. Let us assume that in the year 1920 the positron was discovered as a particle of the same mass as the electron but of opposite charge. It would not require great imagination to believe that somebody would come up with the idea of charge conjugation symmetry, stating that to every particle there exists a charge conjugate particle with the same mass and opposite charge. Such a principle would in time receive irrefutable experimental support in the discovery of antiprotons and of oppositely charged mesons, and would be considered a fundamental law of nature. However, it would be phenomenological and descriptive in character in comparison with our present understanding of the 


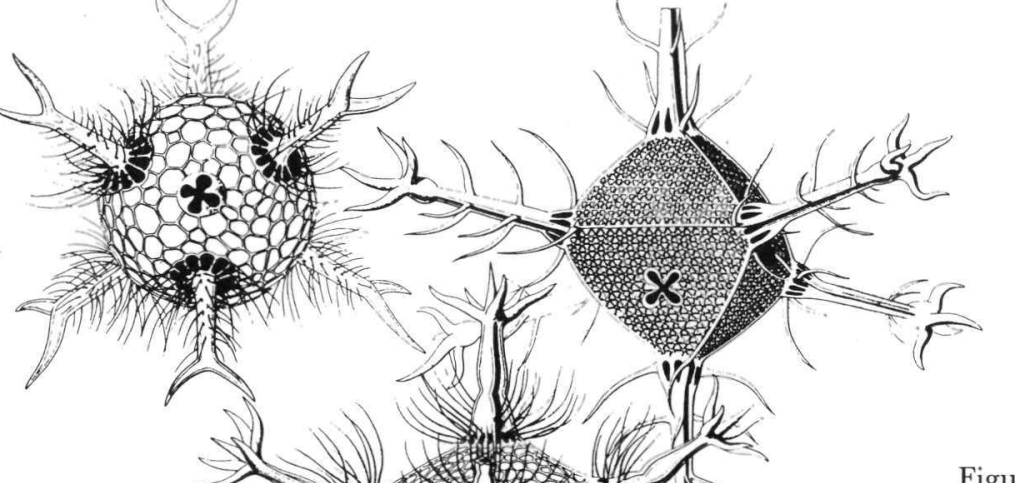

Figure 4. Skeletons of some radiolarians. [From Haeckel, Ernst, and Challenger monograph, Report on the Scientific Results of the Voyage of H.M.S. Challenger, Vol. 18, Pl. 117, H.M.S.O., 1887.]

Figure 5. Ceremonial bronze vessel of type fang i. Smooth grey to green patina. Shang dynasty, China. Dimensions of vessel $=19.1 \times 10.7 \times 7.7$ $\mathrm{cm}$. Collection of the Freer Gallery of Art., Accession No. 54. 13.)

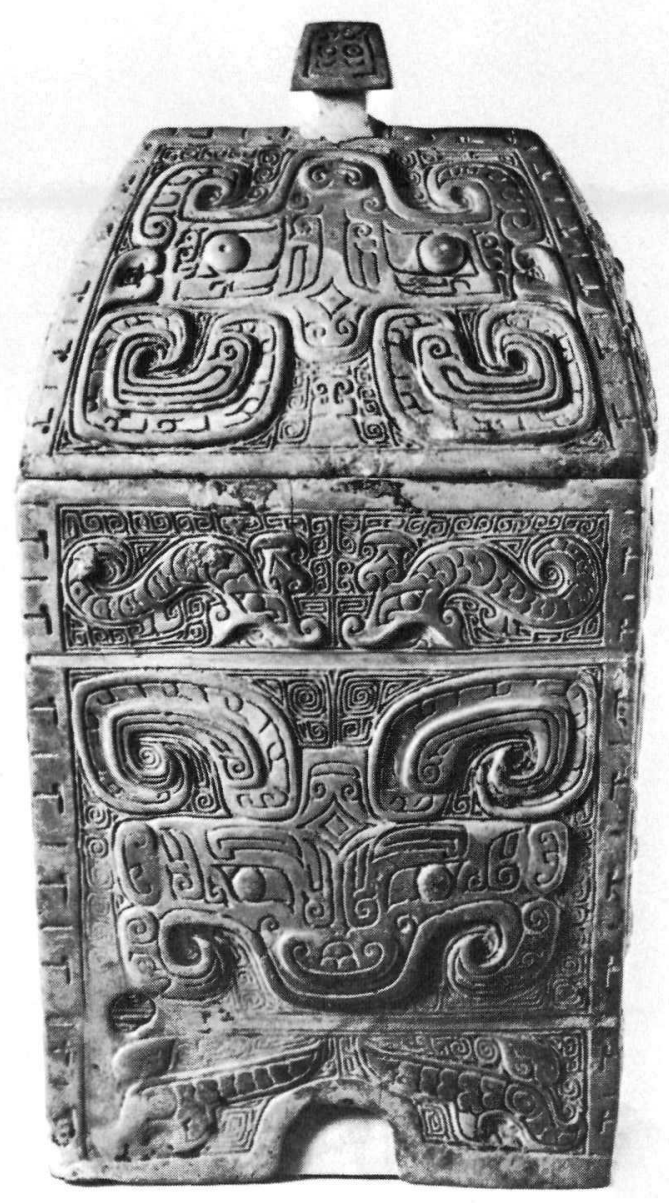




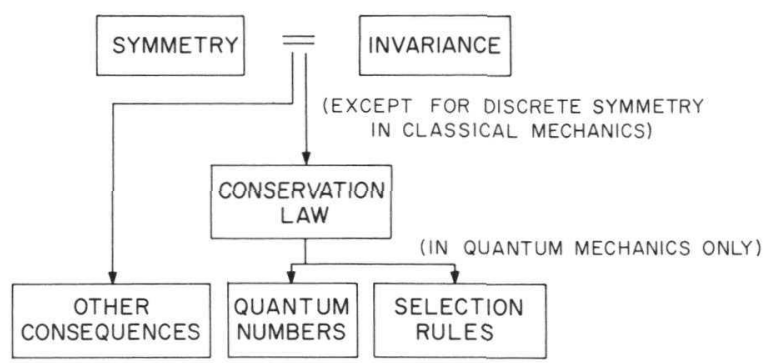

Figure 6. Consequences of symmetry principles. Symmetry of physical laws is also stated as invariance of the laws under symmetry operations. "Other consequences" include, for example, constraints on crystalline structure due to symmetry. [From C.N. Yang, Science 127, 565 (1958).]

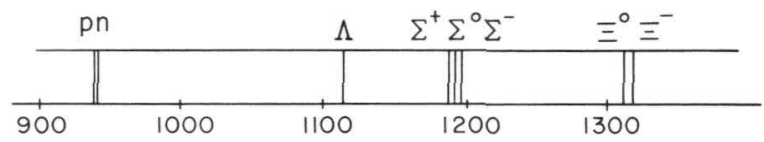

Figure 7. Section of mass spectrum of elementary particles. The clustering of several particles of consecutive charges is typical throughout the spectrum. It is a demonstration of a symmetry called "isotopic spin" symmetry.

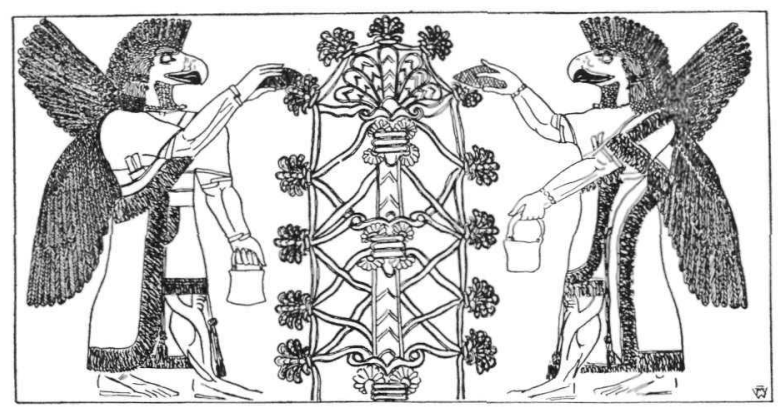

Figure 8. Symmetrical forms with slight deviations from exact symmetry. [From M.H. Swindler, Ancient Painting, p. 45, Yale University Press, 1929.]

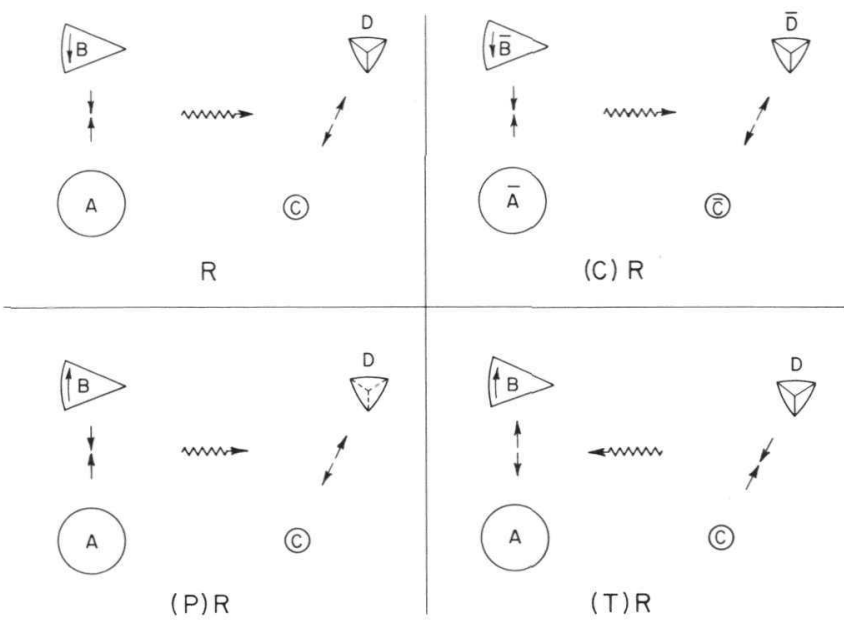

Figure 9. The operations $C, P$, and $T$. The reaction $R$ describes $A+B \rightarrow C+D$ where $A$ and $C$ are spheres, $B$ a spinning cone, and $D$ a tetrahedron. The charge conjugate reaction $(C) R$ has the same kinematics as $R$, but with all particles replaced by antiparticles. $(P) R$ is the reaction $R$ reflected in the plane of the drawing. Notice the reversed spin of $B$ and the reflected tetrahedron $D$. (T)R is the time-reversed reaction of $R$, describing $C+D \rightarrow A+B$. Notice the spin direction of $B$.

\section{Table 1}

Symmetries discussed in elementary particle physics. Geometrical symmetries originate in concepts related to the space-time description of the physical world.
1. Geometrical
Lorentz, $\mathcal{T}, P$
2. Algebraic
$C$
3. Other, exact
4. Other approximate
$Q$, Gauge, $\mathcal{N}, \mathcal{N}_{\text {lepton }}$
5. Crossing
6. $\mathrm{SU}_{6}$, etc.

Table 2

The four classes of forces. Column on right indicates the strengths of the forces. For elementary particles the gravitational forces are very weak and so far experimentally unobserved. Gravitational forces, however, are cumulative and become very important between large bodies. The electromagnetic forces are responsible for most chemical, electric, and magnetic phenomena. The nuclear and weak forces are subjects that have been under intensive study in the last twenty years. They are not yet as well understood as the other two types of forces.
1. Nuclear forces
1
2. Electromagnetic forces
$10^{-2}$
3. Weak forces (decay interactions)
$10^{-13}$
4. Gravitational forces
$10^{-38}$

\section{Table 3}

Experiment directly showing violation of charge conjugation invariance $(C)$. When $\mu^{+}$decays, the $e^{+}$that results is found to be right-handed, i.e., the $e^{+}$spins around its direction of motion as a right-handed screw. If $C$ invariance were to hold, the $C$ operation on the decay of $\mu^{-}$would lead to the decay of $\mu^{-}$into right-handed spinning $e^{-}$(cf. Figure 9) in contradiction with experimental findings.

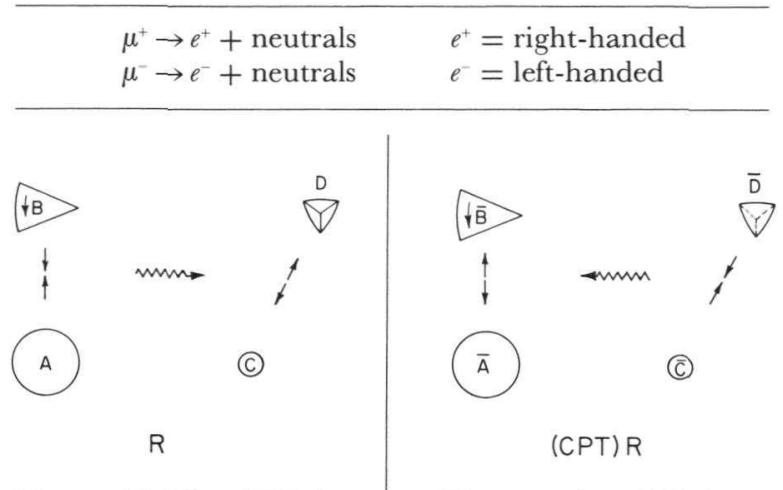

Figure 10. The CPT theorem. The reaction $(C P T) R$ is obtained by applying to $R$ the operations $T, P$, and $C$ in succession. (Each of these operations was defined in Table 2 ). The $C P T$ theorem states that under very general conditions the rates of $R$ and of (CPT) $R$ are, figuratively speaking, the same. See text. 
principle of charge conjugation symmetry. The latter is algebraic in origin and was first theoretically predicted by Dirac's hole theory. It is this deeper understanding that is still lacking in the case of the isotopic spin symmetry.

Before we discuss in more detail the geometrical and algebraic symmetries designated by $C, P$, and $T$, let me mention the fact, especially emphasized by Hermann Weyl, ${ }^{2}$ that oftentimes in art one does not adhere to a strict symmetry. In fact, the existence of a general balance created by symmetrical forms, with minor perturbations causing asymmetry, may create a highly artistic effect. Figure 8 is an illustration taken from Sumerian art showing this principle in operation.

It seems that God in creating the physical world also chose to have certain symmetries largely but not perfectly obeyed. The story of the unfolding of these imperfections in the symmetries has provided much excitement in elementary particle physics in the last 10 years. It turned out that the symmetries $C, P$, and $T$ are obeyed in dominant interactions (i. e., strong and electromagnetic interactions) between elementary particles, while imperfections in these symmetries are observed in "weak interactions." The neat pattern of the division of interactions into four categories with vastly different strengths, as illustrated in Table 2 , is in itself a great mystery. Physicists have learned to live with this pattern, without really understanding it. Perhaps the very origin of the weak interaction has something to do with the observed violation of symmetry. But no one knows.

To describe the symmetries $C, P$, and $T$ we consider a reaction $R$ as illustrated in Figure 9. Corresponding to such a reaction one can imagine the charge conjugate, reflected, and time-reversed reactions denoted by $(C) R,(P) R$, and $(T) R$. Such imagined reactions may or may not be physical processes. If they are, e. g., if $(C) R$ takes place with equal probability as $R$, then one says that the laws governing the process $R$ are invariant under charge conjugation.

An extremely important theorem was proved in the 1950's that under very general conditions the $(C P T)$ conjugate of $R$, illustrated in Figure 10, takes place with equal probability* as $R$ even if $C, P$, and $T$ invariances are individually violated. We can write this symbolically as

$$
(C P T) R \cong R .
$$

*Figuratively speaking. There is a precise statement which we need not elaborate on here.

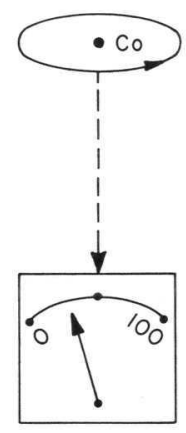

COUNTER
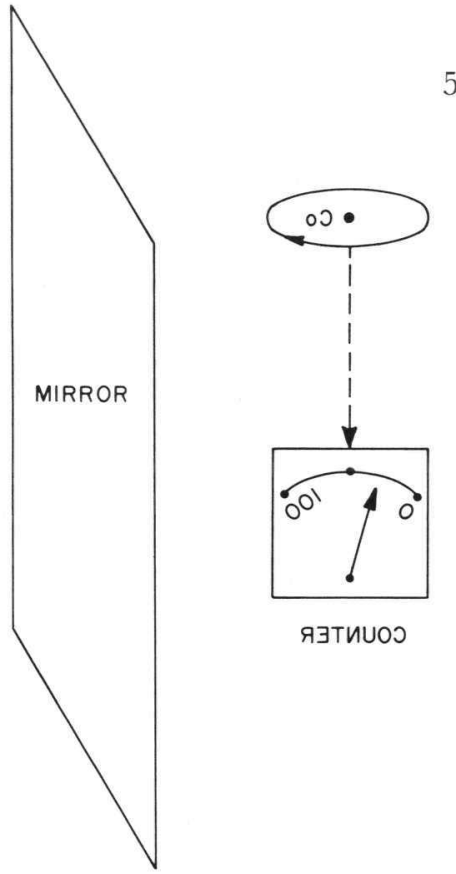

Figure 11. Schematic diagram of the experiment directly showing violation of reflection invariance $(P)$. The experiment, by Wu, Ambler, Hayward, Hoppes, and Hudson, concerned the $\beta$-decay rate of $\mathrm{Co}^{60}$ which was polarized by a current-carrying loop as indicated in the diagram. The mirror image arrangement represented on the right here was found to give a different counting rate. [Schematic diagram from C.N. Yang, Science 127, 565 (1958).]

From this theorem we see that $C P$ invariance implies $T$ invariance, since $C P$ invariance implies

$$
(C P T) R \cong(T) R .
$$

Thus

$$
R \cong(T) R .
$$

Up to 1957 it was believed that $C, P$, and $T$ invariance separately holds for all physical phenomena. That year brought a great shock in the first concrete evidence, through the experiment of Wu, Ambler, Hayward, Hoppes, and Hudson, ${ }^{3}$ that $P$ invariance holds only for strong and electromagnetic interactions and is violated in the weak interactions. The details of the experiment are necessarily complicated. But the essence is simple and can be understood in daily language as shown in Figure 11. What the experiment demonstrated was therefore an unambiguous violation of rightleft symmetry in the $\beta$ decay of cobalt.

The same experiment also demonstrated through some theoretical arguments the violation of $C$ invariance in $\beta$ decay. However, today we can point to a more direct evidence of violation of $C$ invariance through the experiment illustrated in Table 3. 


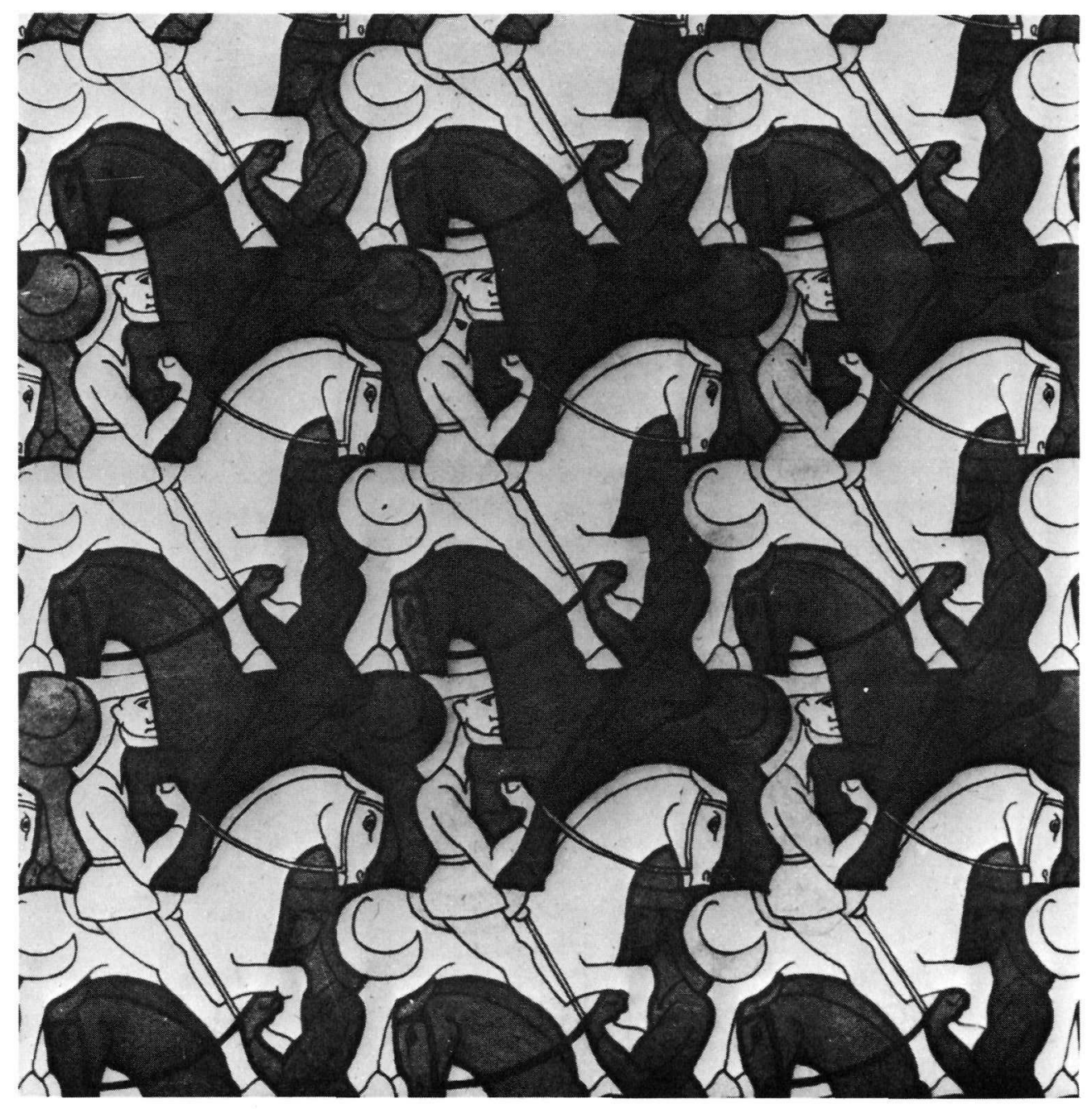

Figure 12. Drawing by M.C. Escher which can illustrate $C P$ invariance. The drawing is different from its mirror image since in the drawing white knights show left arms while in the mirror image white knights show right arms. The " $C P$ " image of the drawing is obtained from the usual mirror image by switching the colors white and black. In the " $C P$ " image white knights show left arms, just as in original drawing. (In this discussion we ignore edge effects, i.e., we imagine the drawing to be infinitely large.) [From C.N. Yang, Elementary Particles, a Short History of Some Discoveries in Atomic Physics, Princeton University Press, 1962].

Figure 13. Possible experiment to demonstrate directly the violation of $C P$ invariance. Decay of $K^{\circ}$ and $\bar{K}^{\circ}$ emitted from sources at the same velocity are observed separately. $K^{0}$ and $\bar{K}^{0}$ are $C P$ images of each other. If present understanding of the experiment of Christenson, Cronin, Fitch, and Turlay is correct, the decays should have drastically different probabilities some distance downstream. Observation of this difference would constitute direct evidence of violation of $C P$ invariance.
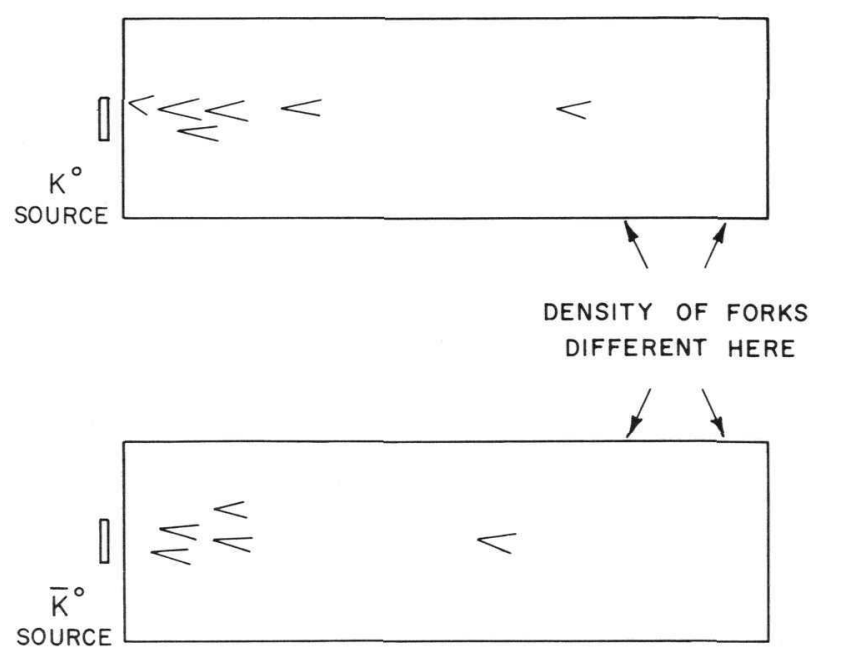
Why is right-left symmetry almost observed, but not quite? Nobody knows the answer to this question. But for a few years after 1957 it was thought that right-left symmetry could still be salvaged through a changed definition of the mirror image process. Combined with the reflection process, one could require a switch from particles to antiparticles. In other words, one could define the "true reflection" process as CP instead of just $P$. It was hoped that physical laws would be invariant under this operation, a hypothesis consistent with the not very accurate experimental data then available. (For example, the experiment of Table 3 does not show violations of $C P$ invariance since the reaction

$$
\mu^{+} \rightarrow \text { right-handed } e^{+}
$$

has as its $C P$ conjugate reaction

$$
\mu^{-} \rightarrow \text { left-handed } e^{-}
$$

and they do have equal rates.) Under the hypothesis, physical laws are invariant under $C P$ and via the $C P T$ theorem also invariant under time reversal, as remarked before. In this way some symmetry is lost, but right-left symmetry is still obeyed in a changed sense. (The changed sense of reflection has been illustrated ${ }^{4}$ in a marvelous drawing by the Dutch artist M.C. Escher reproduced in Figure 12).

However, such a hypothesis was shattered in the summer of 1964 when an experiment done by Christenson, Cronin, Fitch, and Turlay ${ }^{5}$ produced conclusive evidence that $C P$ invariance is also not valid for the weak interactions. The details of this experiment are again rather involved and the inference from it that $C P$ invariance is violated is not quite straightforward. It is, however, possible to conceive of a direct test of the violation of $C P$ invariance as illustrated in Figure 13. The experiment is very difficult because an enormous number of $K$ and $\bar{K}$ particles must be produced. But judging from the rapid stride of experimental physics in the postwar years, it is safe to assume that some day this experiment will be done.
With the violation of $C P$ invariance, it is generally believed on the grounds of the CPT theorem that $T$ invariance is also violated. However, an explicit proof of the violation of $T$ invariance in the weak interactions would still be desirable. It is possible to think of such experiments, but unfortunately none seems feasible in the near future.

If time-reversal invariance is violated, is it necessary to re-evaluate the traditional concepts of statistical physics, in particular the concept of entropy? Fortunately the answer to this question is simple. Entropy belongs to the realm of equilibrium statistical physics, within which time-reversal invariance plays no role. Consequently, although violation of time-reversal invariance allows a distinction to be made in elementary particle weak interactions between the past and the future, such a distinction is unrelated to our daily consciousness of the past and the future. The latter is a result purely of the statistical phenomenon of entropy increase.

Reviewing what we have learned of the symmetry exhibited by elementary particles in their strong interactions and the asymmetry in their weak interactions, one is tempted to ask whether an integrated pattern is in sight. Judging from the beautiful logical perfection and the profound experimental consequences of the successes of symmetry considerations in physics, one is entitled to believe that such a pattern, when it emerges, would transmute the whole enterprise. If no one has yet conceived of such a pattern, it is not because physicists have not tried, but because nature has yet not revealed enough of herself.

\section{REFERENCES}

1. F.R. JAPP, Nature 58, 452 (1898); see also Ibid. 59, 101.

2. H. Weyl, Symmetry, Princeton University Press, 1952.

3. C.S. Wu, E. Ambler, R.W. Hayward, D.D. Hoppes, and R.P. Hudson, Phys. Rev. 105, 1413 (1957).

4. C.N. YAng, Elementary Particles, A Short History of Some Discoveries in Atomic Physics, Princeton University Press, 1962.

5. J.H. Christenson, J.W. Gronin, V.L. Fitch, and R. Turlay, Phys. Rev. Letters 13, 138 (1964). 